\title{
Smart Wheel Chair Navigation System using Brain Computer Interface Model for the People with Mobility Disorder
}

\author{
L. MegalanLeo', K. S. Abishek², K Nikhil Preetham³ ${ }^{3}$ Vedanarayanan.V4, \\ A. Aranganathan ${ }^{5}$, T. Gomathi ${ }^{6}$, Poonguzhali. ${ }^{7}$ \\ 1, 2,3,4,5,6,7 School of Electrical and Electronics Engineering, Sathyabama \\ Institute of Science and Technology, Chennai-600119, Tamilnadu, India
}

\begin{abstract}
Many people around the world suffer by mobility disorders, and most of them rely on power wheelchairs to move around them. However, most patients are not advised to use power wheelchairs because it requires more power and complex interface. In this paper, a Brain Computer Interface application is developed to operate on receiving EEG signals from the brain. Brainwaves are used to control the wheel chair operation in real time. It helps physically challenged people to live a self-supported life. Brain computer interfaces are the systems that read brain wave signals from the human mind and pass it to the physical device with the help of Arduino processor. The signals from the brainwave sensor are processed using Matlab processing. Automation modules can be managed using the brain control interface system. Proposed technique assists and enhances the lifestyle of the disabled people. The goal of the brain-computer interface is to develop a fast and reliable communication between the brain waves of the disabled people and the personal computer.
\end{abstract}

\section{KEY WORDS: BRAIN COMPUTER INTERFACE (BCI), EEG SIGNALS, MOBILITY DISORDERS, BRAIN WAVE COMMUNICATION.}

\section{INTRODUCTION}

Brain Computer interface communicates directly with the human brain and physical devices by transferring the brain signal as command in real time (Ou et al. 2012). Brain Computer Interface (BCI) is the Mind Machine Interface (MMI). MMI is the direct communication path between the brain and the external device. BCIs are often guided by helping, improving, or repairing human cognitive or sensory-motor functions. The BCI Randi field is focused

\section{ARTICLE INFORMATION}

*Corresponding Author: megalanleo@gmail.com

Received 9th Oct 2020 Accepted after revision 29th Dec 2020

Print ISSN: 0974-6455 Online ISSN: 2321-4007 CODEN: BBRCBA

Thomson Reuters ISI Web of Science Clarivate Analytics USA and Crossref Indexed Journal

\section{Clarivate
Analytics}

NAAS Journal Score 2020 (4.31)

A Society of Science and Nature Publication,

Bhopal India 2020. All rights reserved.

Online Contents Available at: http//www.bbrc.in/

Doi: http://dx.doi.org/10.21786/bbrc/13.13/46 primarily on neuroprosthetic applications with the aim of restoring damaged hearing, vision and movement. Electroencephalogram is a measurement of the voltage fluctuations of the brain found in the electrodes of the skull. It is an assessment of the cumulative electrical activity of neurons. Electroencephalography (EEG) is the most studied non-invasive interface, mainly due to its excellent temporal resolution, ease of use, portability and low setup cost.

$\mathrm{BCI}$ system receives input from the human mind with the help of acquisition device. It is processed using the signal processing tool. Processed output is given to classifier; it makes a decision based on the input it receives. It will be given to the output device interface. Gamma, beta, alpha, theta and Delta are the different states of brain wave which frequency value differs among different states. Amplitude value also deviates among different state. This differentiation helps to design a brain controlled interface. The neurosky Mindset is a brain wave sensing

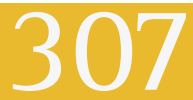


headset that uses a medical grade probe to capture brain patterns and translate them into things you can do with a computer. Exploring neuroscience has greatly expanded our insight into the electrical signals emitted by the cerebrum, especially neurons. Examples of these electrical signals and frequency enzymes can be measured by inserting a sensor into the skull. The neurosky Think Gear Innovation Mindset contains simple electrical signals, usually excluding them as brain waves that make them a computerized symbol to access predictions for restorative applications. The Think Gear is a novelty in every neuroscience that empowers the gadget to interface with the clothing's brain waves. It includes a sensor that touches the forehead, reference points on the ear pad, and an on-board chip that processes all the data.

Older people, injured people and the disabled people should be supported with the technology. Robot can help them by following the instructions they pass (Wolpaw \&t McFarland 2004). In 2014, United States National spinal cord injury statistical center (NSCISC) report says that 276,000 people are living with spinal cord injury (Cao et al. 2014; Boughner \& Durfee 2014; Perrin et al. 2010). In 2002, Arnaud Delorme et all developed an open source toolbox to analyze EEG signals (Delorme Ct Makeig 2004). In 2001, furtscheller et all developed a communication model to communicate the brain signals with the imaginary motor direction. It matches the brain signal to rotate the motor in left, right, forward and backward (Pfurtscheller \& Neuper 2001). In 2002, J. del R. Millan et all proposed a neural classifier to distinguish the brain signals and achieved an accuracy of $70 \%$ (del R Millan et al. 2002). In 2013, Luzheng Bi et all designed a brain controlled robots to support the disabled person in the functional way.

Detailed analysis is given in the perspective and operational modes of robots. Challenges and issues in the brain controlled interface are also discussed (Bi et al. 2013). In 2014, T Kaleswapnil et al. controlled a robot movement with the EEG signals. Interface uses FPGA Kit with mat lab environment (Kale Swapnil et al. 2014). In 2015, kamalesh et al. designed a robot to help handicapped people. It uses the beta wave to communicate with remote device. In 2014, Siliveru Ramesh, designed a robot which is controlled using brainwaves and it uses Bluetooth technology to communicate with them. In 2006,Katarzyna Blinowska et all explained the EEG signals, its generation and how it is measured (Blinowska \& Durka 2013). In 2013, Daniel Göhring et all, presented an approach to control the car with the brain signals. They analyzed the outcome by doing different experimentation. In 2011, luna wrote an article which explained the technique to control the machine using brain waves (Nithya \&t Ramesh 2020).

In 2004, Fernando et all described the functional technique to map the brain signals. In 2013, kottimalai et all proposed a brain wave classification technique using neural network and also they analyzed the patterns of the brain signals. In 2015, Seungchan Lee, designed an active dry electrodes to capture the brain signal for designing BCI System (Kottaimalai et al. 2013).In 2011, Vangu Kitoko et all evaluated the performance of dry electrode with bristle to record the EEG data (Satpathy et al. 2020). In 2004, Millan et all controlled an advanced robot using the EEG signals. They achieved a performance ratio of 74\% (Lee et al. 2015). In 2017, Ganesan et all developed a robot to manage the warehouse products which uses motor controlled by processor (Kitoko et al. 2011). In 2019, Leo et all developed an IoT based traffic management system where they uses sensors with GSM technology to pass the information to the centralized server (Millan et al. 2004; Rajendrakumar \& Parvati 2019; Prabu et al. 2019).

Figure 1.1: Brain MRI where stroke regions are present

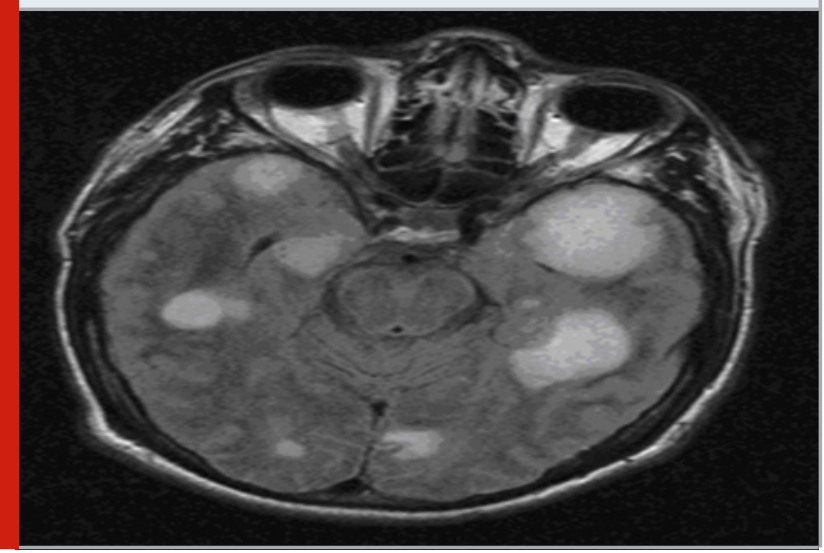

Figure 1.2: Functional Block Diagram of Brain controlled Wheel Chair

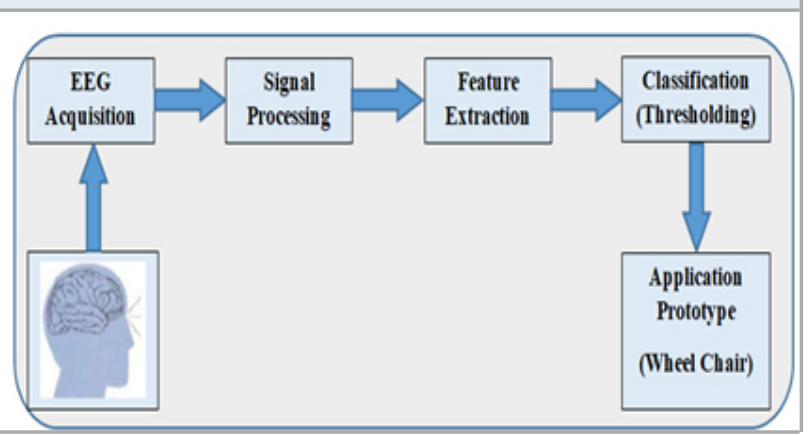

Experimental Methods

Neurosis device placed on the skull collects the brain waves from the human. These waves are analyzed and processed in the matlab environment. Preprocessed signals are given to feature extractor which is used to extract the important feature from the preprocessed signals. Extracted features are given to classifier module. Classifier module makes a decision and passes the commands to arduino Mega 2560. Arduino device is used as a wheel chair controller. The tool is programmed by matlab and controls forward, backward, left and right movements based on the concept of eye blinks. The EEG sensor available in the neurosis device separates and amplifies small electrical voltages produced by the mind cells (neurons). Typical frequency values of EEGs range from 1 to $40 \mathrm{~Hz}$. 
The EEG sensor records the "raw" EEG flag, the possibility of constantly switching between the positive and negative anode, and the product forms that flag the collection of computerized channels to the recorded flag with the end goal in mind. The EEG flag has different frequency groups. It includes delta band frequency range is $(0.5-4 \mathrm{~Hz})$ which is also called as relaxation band, theta band frequency range is $(4-8 \mathrm{~Hz})$ which is also called as laziness band, alpha band frequency range is $(8-13)$ $\mathrm{Hz}$ which is also called as preparedness and innovation band and beta band (13-25Hz). Changes in the alpha band imply decrease in fatigue energy variation and an increase in the theta, delta band. Brain wave sensors are used to capture the brainwave signals and it is transmitted to the signal processing unit with the help of Bluetooth. Here RAW data generated by sensors are transmitted using Bluetooth module available in neurosis device. In the wheel chair Bluetooth receiver receives the signal. It will be given to signal processing unit which is built on mat lab environment.

Acquired EEG signals are given to the matlab interface. Think gear is a mat lab application which is used to record and decode the raw data collected from the acquisition device. Mind wave sensor placed in the skull captures the brain waves variation and it is given to the think gear module. Think gear module preprocess the acquired data and amplify the signal strength. Preprocessed signals are given to feature extraction module. It extracts the important features from the signal. Classifier generates the output based on the extracted features. Classifier output is given to arduino board which generates control the wheel chair to move forward, backward, left and right. Materials and Methods

Figure 1.3: Prototype model for Brain controlled wheel chair

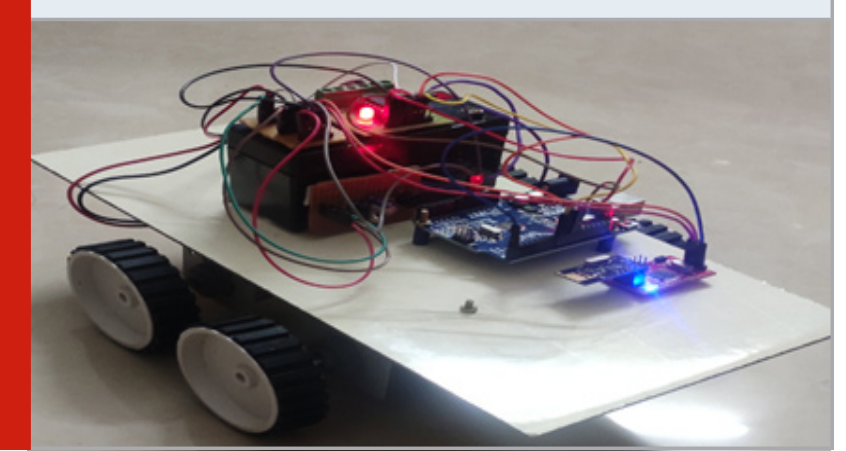

RESULTS AND DISCUSSION

Prototype model for controlling the wheel chair using brain waves are designed using the arduino processor and signals are processed in the matlab environment. Prototype model is shown in figure 1.2. Brain wave signals are captured using the sensors available in the neurosis device. Captured EEG signals are the raw data. Raw data are transferred to interface module with the help of Bluetooth. Here the information is transferred from the head device to the wheel chair using wireless technology. Wheel chair has a Bluetooth receiver which receives the signal transmitted by the neurosis device. Received raw data are given to matlab environment where Think gear application is used. Think gear can handle the EEG signals and analyzed it.

Brain wave signals are varying in energy based on the action performed by the user. For example, if a user blinks eyes for single time, brain wave signal energy is less. If the same person blinks eyes for two times, brain wave energy signal is more compared to the previous. Brain wave signal for blinking the eyes for a time is shown in figure 1.3. EEG Raw data are processed and the feature is extracted. Features are the energy value of the signal. The main code for reading, analyzing, classifying and commanding brain waves is written in MATLAB. Mat lab code is used to read the brain waves attention Level and Eye Blink Strength. Classifier generates the control signal and it will be passed to the Arduino board where program to control the motors are written. Classifier gives the output as A when eye blinks for a time. Output B will be generated if the eyes blink for two times.

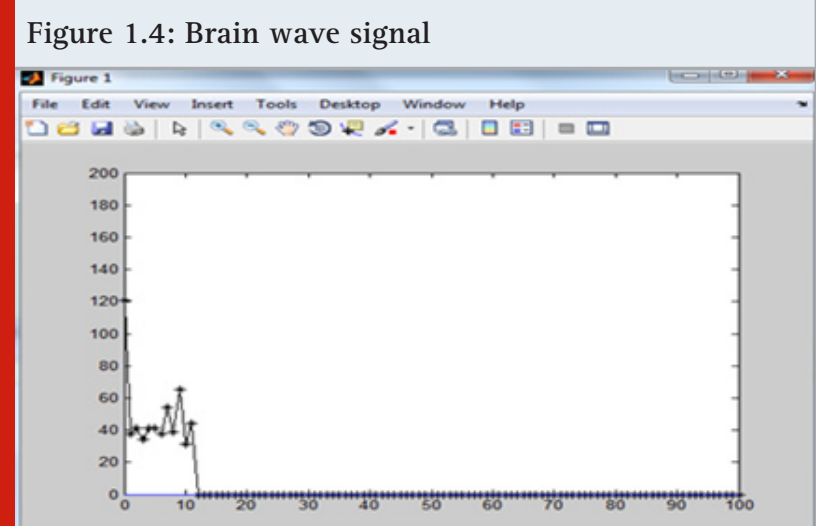

Figure 1.5: Arduino Code for controlling the motors

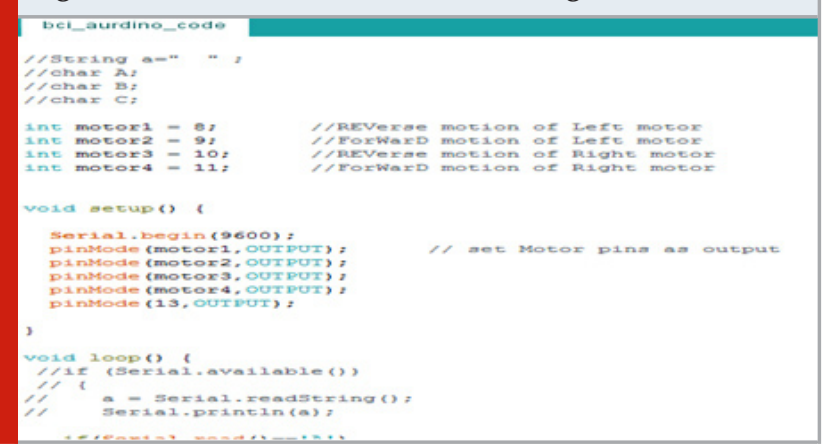

It gives output $\mathrm{C}$ when head moves from left to right. It gives output D when head moves from right to left. Variation in the EEG Value captured at the feature extraction module. Arudino program uses to control the motors connected with the wheels of the chair. If the classifier output is A then Arduino generates the control signal to the motor to move the wheel chair in the forward direction. For the classifier output B, Arduino generates the control signal to the motor to move the wheel chair in the reverse direction. Received input is C then Arduino issue commands to rotate the wheel to 
turn towards right direction. If the classifier output is $\mathrm{D}$ then Arduino generates the control signal to rotate the wheel to turn towards left direction. If the classifier output is E, then arduino stops the movement of wheel. It remains in idle position. In Arduino code is built for controlling the Robot. Arduino (ATMega328) is used as wheel chair controller. Fig.1.4 shows the sample code, with character "A" for FORWARD, "B" for BACKWARD, "C" for RIGHT "D" for LEFT and "E" for STOP.

\section{CONCLUSION}

Brain controlled wheel chair is designed to help the people who uses the wheel chair. It can help them to control the wheel chair without using physical energy. Wheel chair is controlled by brain waves. Eye blinking and head movement generates unique brain waves. It helps to control the device which is connected using Bluetooth. Wireless connectivity ensures free physical action of the disabled people. Designed prototype model is designed and tested. It works well and wheel chair is also controlled towards the command issued through the brain waves. This technique can be used to control remote devices effectively. Cerebrum Wave Sensor collects EEGbased mind markers of various frequency and amplitude, transmits these gestures to the level splitter section via Bluetooth media. The main difference between braincontrolled frameworks and other personal-controlled gadgets is that these frameworks require a high level of safety because it helps the disabled people. Research on Mind Control Frameworks has been very successful. Further operational and practice of this finding leads to the development of mechanisms that can be used by clients and consequently increases their expertise, independence and personal satisfaction. A standardized assessment methodology is needed to test the innovation functions of the BCI system. It can be used in medical applications to monitor the disabled and aged people. Monitor alertness, coma and brain death.

\section{REFERENCES}

Bhoi A. K., Mallick P. K., Liu C. M., \&t Balas V. E.: Bioinspired Neurocomputing, Springer Nature, (2021).

Bi L., Fan X.A., and Liu Y.: EEG-based brain-controlled mobile robots: a survey. IEEE transactions on humanmachine systems, vol. 43, pp.161-176, (2013).

Blinowska K., and Durka P.: Electroencephalography (eeg). Wiley encyclopedia of biomedical engineering, (2006).

Boughner K.J., and Durfee W.K.: Preliminary design of an energy storing orthosis for providing gait to people with spinal cord injury. International Conference of the IEEE Engineering in Medicine and Biology Society, pp. 2581-2584, (2014).

Cao Y., Massaro J.F., Krause J.S., Chen Y., and Devivo M.J.: Suicide mortality after spinal cord injury in the United States: injury cohorts analysis. Archives of physical medicine and rehabilitation, vol. 95, pp.230235, (2014).

da Silva F.L.: Functional localization of brain sources using EEG and/or MEG data: volume conductor and source models. Magnetic resonance imaging, vol. 22, pp.1533-1538, (2004).

del R Millan J., Mouriño J., Franzé M., Cincotti F., Varsta M., Heikkonen J. and Babiloni F.: A local neural classifier for the recognition of EEG patterns associated to mental tasks. IEEE transactions on neural networks, vol. 13, pp.678-686, (2002).

Delorme A., and Makeig S.: EEGLAB: an open source toolbox for analysis of single-trial EEG dynamics including independent component analysis. Journal of neuroscience methods, vol. 134, pp.9-21, (2004).

Ganesan P., Sajiv G. and Leo L.M.: Warehouse management system using microprocessor based mobile robotic approach. In 2017 Third International Conference on Science Technology Engineering \& Management (ICONSTEM), pp. 868-872, (2017).

Göhring D., Latotzky D., Wang M., and Rojas R.: Semi-autonomous car control using brain computer interfaces. In Intelligent Autonomous Systems, vol.12, pp. 393-408, (2013).

Kale Swapnil T., MahajanSadanand P., and RaksheBalu G.: Robot Navigation control through EEG Based Signals. International Journal of Engineering and Computer Science, vol. 3, pp.5105-5108, (2014).

Kitoko V., Nguyen T.N., Nguyen J.S., Tran Y., and Nguyen H.T.: Performance of dry electrode with bristle in recording EEG rhythms across brain state changes. In 2011 Annual International Conference of the IEEE Engineering in Medicine and Biology Society, pp. 59-62, (2011).

Kottaimalai R., Rajasekaran M.P., Selvam V. and Kannapiran B.: EEG signal classification using principal component analysis with neural network in brain computer interface applications. In 2013 IEEE International Conference ON Emerging Trends in Computing, Communication and Nanotechnology (ICECCN) pp. 227-231, (2013).

Lee S., Shin Y., and Lee H.N.: Design of active dry electrodes and its evaluation for EEG acquisition. In 2015 International Conference on Information and Communication Technology Convergence (ICTC), pp. 560-562, (2015).

Leo L.M., Tummala K., and Raju R.P.: IoT based Centralized System for Emergency Vehicle Passing with Authentication. In 2019 International Conference on Communication and Electronics Systems (ICCES), pp. 1543-1547, (2019).

Luna, P.: Controlling machines with just the power of thought. The Lancet Neurology, vol. 10, pp.780-781, (2011).

Mallick P. K., Balas V. E., Bhoi A. K., and Chae G.-S. (Eds.): Cognitive Informatics and Soft Computing: Proceeding of CISC 2019, Vol. 768, (2020).

Mallick P. K., Balas V. E., Bhoi A. K., and Zobaa A. F. (Eds.): Cognitive Informatics and Soft Computing: Proceeding of CISC 2017, Vol. 768, (2019). 
Millan J.R., Renkens F., Mourino J., and Gerstner W.: Noninvasive brain-actuated control of a mobile robot by human EEG. IEEE Transactions on biomedical Engineering, vol. 51, pp.1026-1033, (2004).

Mishra S., Tripathy H. K., Mallick P. K., Bhoi A. K., and Barsocchi P.: EAGA-MLP-An Enhanced and Adaptive Hybrid Classification Model for Diabetes Diagnosis. Sensors, vol. 20, pp. 4036 (2020).

Nithya V., and Ramesh G.P.: Wireless EAR EEG Signal Analysis with Stationary Wavelet Transform for Co Channel Interference in Schizophrenia Diagnosis. Intelligent Systems Reference Library, vol. 172, (2020).

Ou C.Z., Lin B.S., Chang C.J., and Lin C.T.: Brain computer interface-based smart environmental control system. International Conference on Intelligent Information Hiding and Multimedia Signal Processing, pp. 281-284, (2012).

Perrin X., Chavarriaga R., Colas F., Siegwart R., and Millán J.D.: Brain-coupled interaction for semiautonomous navigation of an assistive robot. Robotics and Autonomous Systems, vol. 58, pp.1246-1255, (2010).

Pfurtscheller G., and Neuper C.: Motor imagery and direct brain-computer communication. Proceedings of the IEEE, vol. 89, pp.1123-1134, (2001).

Prabu, S., Lakshmanan, M. and Mohammed, V.N., 2019. A multimodal authentication for biometric recognition system using intelligent hybrid fusion techniques. Journal of medical systems, 43(8), pp.1-9.

Rajendrakumar, S. and Parvati, V.K., 2019, January. Automation of irrigation system through embedded computing technology. In Proceedings of the 3rd International Conference on Cryptography, Security and Privacy (pp. 289-293).

Satpathy R.B., and Ramesh G.P.: Advance Approach for Effective EEG Artefacts Removal. Intelligent Systems Reference Library, vol.172. (2020).

Siliveru Ramesh K.H., and Chaitanya J.K.: Brainwave controlled robot using bluetooth. International Journal of Advanced Research in Electrical, Electronics and Instrumentation Engineering, vol. 3, pp.11572-11578, (2014).

Solanki K.H., and Pujara, H.: Brainwave controlled robot. International Research Journal of Engineering and Technology (IRJET), vol. 2, (2015).

Wolpaw J.R., and McFarland D.J.: Control of a twodimensional movement signal by a noninvasive brain-computer interface in humans. Proceedings of the national academy of sciences, vol. 101, pp.1784917854, (2004). 